Article

\title{
Integral Field Spectroscopy of Planetary Nebulae with MUSE
}

\author{
Jeremy R. Walsh 1,*(D) and Ana Monreal-Ibero 2,3 (iD \\ 1 European Southern Observatory, 85748 Garching, Germany \\ 2 Instituto de Astrofísica de Canarias (IAC), E-38205 La Laguna, Tenerife, Spain; amonreal@iac.es \\ 3 Dpto. Astrofísica, Universidad de La Laguna, E-38206 La Laguna, Tenerife, Spain \\ * Correspondence: jwalsh@eso.org
}

Received: 27 February 2020; Accepted: 29 March 2020; Published: 3 April 2020

\begin{abstract}
The Multi-Unit Spectroscopic Explorer (MUSE) is a large integral field unit mounted on the ESO Very Large Telescope. Its spatial (60 arcsecond field) and wavelength (4800-9300 $\mathrm{A}$ ) coverage is well suited to detailed imaging spectroscopy of extended planetary nebulae, such as in the Galaxy. An overview of the capabilities of MUSE applied to Planetary Nebulae (PNe) is provided together with the specific advantages and disadvantages. Some examples of archival MUSE observations of PNe are provided. MUSE datacubes for two targets (NGC 3132 and NGC 7009) are analyzed in detail, and they are used to show the advances achievable for planetary nebula studies. Prospects for further MUSE observations of PNe and a broader analysis of existing datasets are outlined.
\end{abstract}

Keywords: optical spectroscopy; integral field spectroscopy; planetary nebulae; emission lines; physical conditions; abundances; kinematics

\section{Overview of MUSE}

The Multi-Unit Spectroscopic Explorer (MUSE) is a large field-of-view $\left(\sim 60 \times 60^{\prime \prime}\right)$ optical integral field spectrometer mounted on the European Southern Observatory (ESO) Very Large Telescope (VLT), currently on Unit Telescope 4 (Yepun). The field is divided into 24 slices, and each is sent to a separate integral field unit (IFU) that divides the sub-field into 48 mini slits, which are all fed to one of the 24 identical spectrometers [1]. Each spectrometer module is equipped with a volume phase holographic grating covering the full wavelength range $4600-9300 \AA$ and imaged by an EEVdeep depletion, anti-reflection-coated $4 \mathrm{k} \times 4 \mathrm{k}$ Charge Coupled Detector (CCD). Details of the instrument can be found on the ESO MUSE web page (http:/ / www.eso.org/sci/facilities/paranal/instruments/ muse/inst.html) and in the MUSE Instrument Manual [2].

Although the instrument format is fixed in terms of the number of slicers and spectrographs, there are some options for feeding the field from the VLT to the instrument and one wavelength range choice. Table 1 lists the various options. In Wide Field Mode (WFM), the full field of $300 \times 300$ pixels, each $0.20 \times 0.2^{\prime \prime}$, is covered. Using the VLT Deformable Secondary Mirror (DSM), a Ground Layer Adaptive Optics (AO) feed is available, which provides the same field, but an improved image quality (typically $\times 2$ ensquared energy, but depending on atmospheric conditions). Higher spatial resolution is achievable with laser tomographic AO, and the field size is reduced to 7.5" (Narrow Field Mode (NFM)). It should be noted that, since a sodium laser is employed for the AO modes, the spectral region around the Na I doublet ( $58905896 \AA$ ) is blocked by a filter. In both WFM and NFM, an alternative wavelength range can be assessed (called extended mode), which shifts the blue cut-off from 4800 to $4650 \AA$; however, this mode suffers second order contamination at wavelengths above $8000 \AA$. Most of these modes have been employed for MUSE observations of PNe. 
Table 1. The Multi-Unit Spectroscopic Explorer's (MUSE) instrumental parameters. AO, Adaptive Optics.

\begin{tabular}{llll}
\hline Mode & Field $\left.\mathbf{(}^{\prime \prime}\right)$ & Pixel $\left.\mathbf{( \prime \prime}^{\prime \prime}\right)$ & Image Quality \\
\hline Wide Field Mode (WFM) & $59.9 \times 60.0$ & 0.20 & Native seeing \\
Wide Field Mode + AO & $59.9 \times 60.0$ & 0.20 & 2 $\times$ encircled energy \\
Narrow Field Mode (NFM) & $7.42 \times 7.43$ & 0.025 & 55-80 milli-arcseconds \\
\hline Spectral mode & Wavelength range $(\AA)$ & Resolving power & Field mode \\
\hline Normal (N) & $4800-9300$ & $1770-3590$ & WFM \\
Normal (N) & $4800-5780,6050-9300$ & $1740-3450$ & NFM \\
Extended (E) & $4650-9300$ & $1770-3590$ & WFM \\
Extended (E) & $4650-5760,6010-9300$ & $1740-3450$ & NFM \\
\hline
\end{tabular}

\section{Suitability of MUSE for PNe}

Planetary nebulae are extended emission and continuum nebulae ionized by a central hot star, mostly representing the shell of gas ejected during the previous Asymptotic Giant Branch (AGB) phase of the evolution of a $0.8-8.0 \mathrm{M}_{\odot}$ star. PNe in the Galaxy present projected sizes from fractions of an arcsecond for compact and/or distant PNe to almost half a degree, making them good targets for IFU observation (e.g., [3]). MUSE with its large IFU field can be considered well matched to spectral imaging of a range of Galactic PNe. For nebulae where the emission outskirts of the field are contained within the MUSE field, direct sky removal is facilitated; nebulae much larger can be effectively sky subtracted using an offset sky exposure. Details of the compact structures in larger nebulae, or more compact $\mathrm{PNe}$, are well accessible to the narrow field mode. The native spaxel size of $0.20^{\prime \prime}$ is matched to sampling good Paranal seeing $\left(\sim 0.5^{\prime \prime}\right)$, and smaller scale structures can be imaged in detail; while NFM competes with Hubble Space Telescope imaging to the $0.1^{\prime \prime}$ level.

The spectral coverage of MUSE provides a good sampling of the rich optical emission spectrum consisting of recombination and collisionally excited lines, which have been observed over many decades and have contributed a substantial literature on the lines, their parent atoms and ionization mechanisms, and their use as diagnostics of physical processes (e.g., [4-6]). The applicability of diagnostic line ratios for dust extinction, electron density $\left(N_{\mathrm{e}}\right)$, and temperature $\left(T_{\mathrm{e}}\right)$ measurement and for abundance determination, refined through observations of $\mathrm{PNe}$, have been applied to ionized regions in galaxies at the highest observed redshifts. The MUSE coverage to $9300 \AA$ allows the Paschen continuum jump of ionized $\mathrm{H}$ (and ionized $\mathrm{He}$ ) at $\sim 8210 \AA$ [7] to be measured (although compromised in extended mode by second order contamination), providing another temperature and density probe distinct from those of the emission lines.

The wavelength compass does however bring some disadvantages compared to full optical (3500-10,000 $\AA$ ) and higher resolution spectroscopy:

- the spectral Resolving power $(\mathrm{R})$ is relatively low ( 1800-3600; see Table 1), thus not sampling the emission lines at the optimal value of two times their intrinsic (thermal and Doppler broadened) width $(R>10,000)$. On multiple sampled spectra, some restoration is of course possible to assess line blends below $3-4 \AA$ separation;

- Important diagnostic lines blueward of $4650 \AA$ are not included, including the [O II] doublet $(3726,3729 \AA)$ for $N_{\mathrm{e}}$ measurement, [Ne III] lines, and the auroral [O III] $4363 \AA$, which, by the ratio to the $\left[\mathrm{O}\right.$ III] $4959,5007 \AA$ lines, provides $T_{\mathrm{e}}$ for the higher ionization medium. Alternative, less bright diagnostic lines are available in the MUSE range, involving less major coolants of the gas,

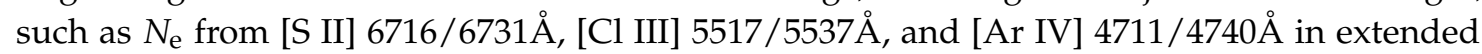

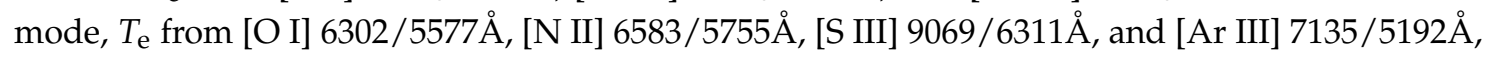
thus partially compensating for the absence of the "classical" $\mathrm{O}^{++}$diagnostic, which samples the bulk ionization component for all but the lowest ionization PNe; 
- The bright and important (at least for higher ionization PNe) He II 4686 MUSE normal mode (but included in extended mode). This absence is partially compensated by the He II $5411 \AA$ line, although $\sim 10$ times fainter;

- The $[\mathrm{O}$ II] $] 7319,7320 \AA$ and $7329,7330 \AA$ doublet is much fainter than the blue [O II] $3726,3729 \AA$ lines, but can be used for the determination of the $\mathrm{O}^{+}$ionic fraction;

- Relatively few Optical metal Recombination Lines (ORLs) are covered in normal mode (mostly N), and while the $4650 \AA ̊$ region important for the brightest O II recombination lines is just included in extended mode, no Ne ORLs are available.

\section{Archival MUSE Observations of PNe}

Some 20 PNe have been observed with MUSE to data at various phases of the instrument commissioning and also in guest observer (open) time. Table 2 lists the PNe observed with an indication of the mode(s) employed. Data for only two PNe (NGC 3132 and NGC 7009; see the next section) have been published to date, reflecting the large analysis task associated with these rich datasets encompassing hundreds of detectable emission lines per spaxel.

Table 2. Multi-Unit Spectroscopic Explorer (MUSE) archival observations of planetary nebulae. Comm., Commissioning.

\begin{tabular}{|c|c|c|c|c|}
\hline Target & Designation & $\begin{array}{l}\text { MUSE } \\
\text { Mode }\end{array}$ & Obs.Type & Notes \\
\hline NGC 6369 & PNG002.4 +05.8 & WFM AO, N & Comm. & \\
\hline M 1-42 & PNG $002.7-04.8$ & WFM, E & $\mathrm{GO}$ & ESO Proposal 097.D-0241 \\
\hline Hf $2-2$ & PNG $005.1-08.9$ & WFM, E & GO & 097.D-0241 \\
\hline GJJC 1 & PNG $009.8-07.5$ & NFM, N & Comm. & IRAS 18333-2357 \\
\hline Sa 3-151 & PNG $033.2-01.9$ & NFM, N & Comm. & \\
\hline NGC 6778 & PNG $034.5-06.7$ & WFM AO, N & Comm. & \\
\hline NGC 7009 & PNG $037.7-34.5$ & WFM, N & SV & Walsh et al. $(2016,2018)$ \\
\hline NGC 7009 & PNG $037.7-34.5$ & WFM, E & $\mathrm{GO}$ & 097.D-0241 \\
\hline NGC 7009 & PNG $037.7-34.5$ & NFM, N & Comm. & \\
\hline Abell 46 & PNG $055.4+16.0$ & WFM, E & $\mathrm{GO}$ & 097.D-0241 \\
\hline IC 418 & PNG $215.2-24.2$ & WFM, N & Comm. & \\
\hline NGC 3242 & PNG $261.0+32.0$ & WFM, E & $\mathrm{GO}$ & 097.D-0241 \\
\hline NGC 2818 & PNG $261.9+08.5$ & WFM, N & Comm. & \\
\hline NGC 3132 & PNG $272.1+12.3$ & WFM, N & Comm. & Monreal-Ibero and Walsh (2020) \\
\hline IC 2501 & PNG $281.0-05.6$ & WFM, N & Comm. & \\
\hline NGC 4361 & PNG $294.1+43.6$ & WFM, N & Comm. & \\
\hline NGC 5189 & PNG $307.2-03.4$ & WFM, N & Comm. & \\
\hline IC 4406 & PNG $319.6+15.7$ & WFM, N & Comm. & Type I PN; see Figure 1 \\
\hline $\mathrm{Mz}-3$ & PNG $331.7-01.0$ & NFM, N & Comm. & \\
\hline CPD-56 8032 & PNG $332.9-09.9$ & NFM, N & Comm. & He 3-1333 \\
\hline NGC 6153 & PNG $341.8+05.4$ & WFM, E & $\mathrm{GO}$ & 097.D-0241 \\
\hline NGC 6563 & PNG $358.5-07.3$ & WFM AO, N & Comm. & See Figure 1 \\
\hline
\end{tabular}

Many PNe were observed as part of the Commissioning (Comm.) of the instrument at various stages (Comm. entries in Table 2), reflecting the fact that they make excellent press release images and enable the capabilities of the instrument to be thoroughly exercised (extended and point sources, line and continuum spectra with many emission lines, some with fixed ratios). Figure 1 shows images from the ESO Release (eso1724 https:/ / www.eso.org/public/news/eso1724/) of some of these PNe observed with WFM and GLAO, well illustrating the quality of the imaging data that can be extracted from the position-wavelength reduced cubes. IC 4406 is a Type I PN(high He, N abundance arising from a higher mass progenitor star [8]) with strong bipolar symmetry; the color image, a combination of the emission in the lines of [O III], [O I], $\mathrm{H} \alpha,[\mathrm{N} \mathrm{II}]$, and [S II], shows the fine scale (dust) extinction features throughout the nebula. In contrast, NGC 6563 is an elliptical shell PN with a classical inner 
high ionization zone and lower ionization shell, projected on a dense stellar field; NGC 6563 shows a fragmented CO emission shell [9].
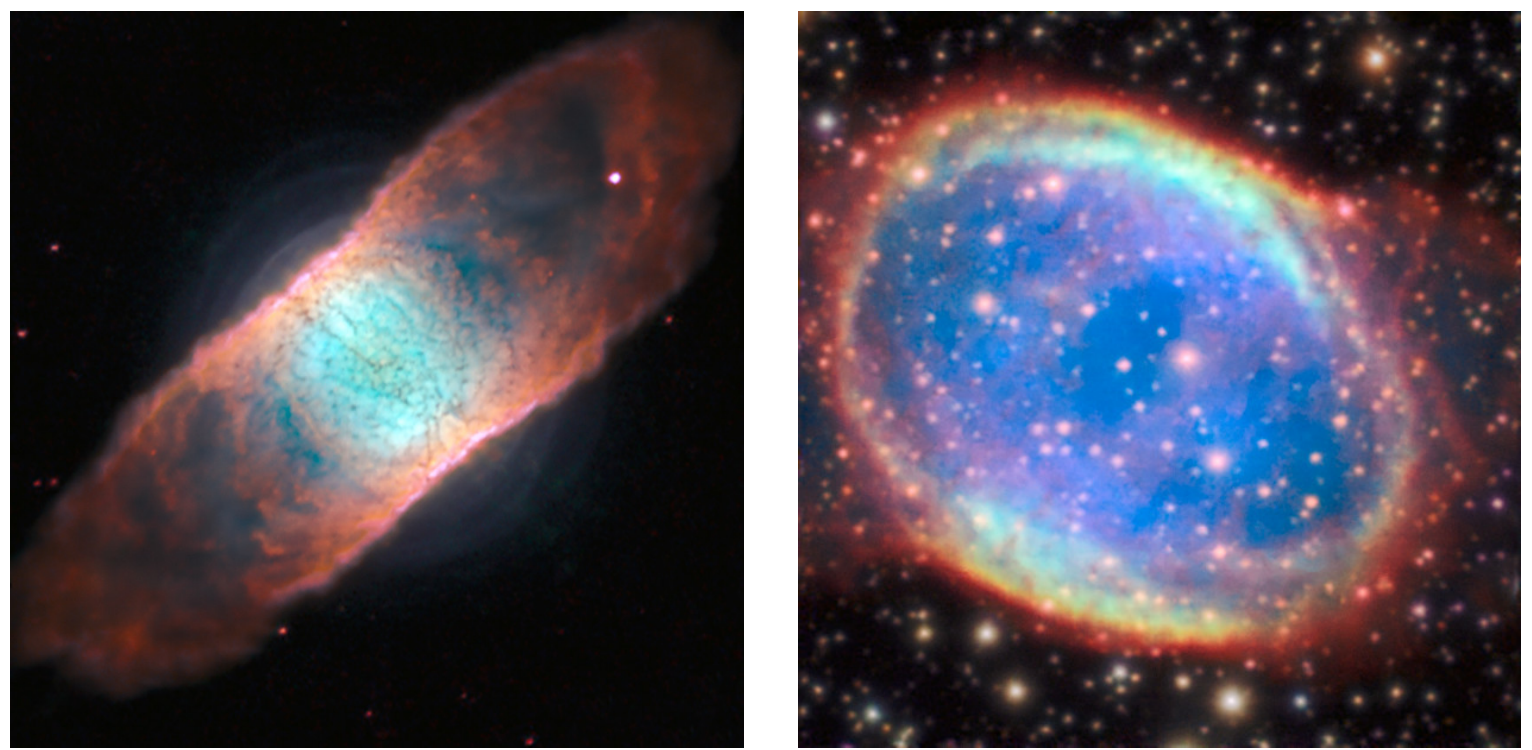

Figure 1. Left: ESO press release images (eso1724) of the bipolar Galactic PN IC 4406 (left, image size $83 \times 83^{\prime \prime}$, north to upper left, east to lower left) and the elliptical PN NGC 6563 (right, image size $62 \times 62^{\prime \prime}$, north to the top), both taken with MUSE as part of the commissioning of the wide field mode with (ground layer) AO correction. The emission line images are color coded according to: [O III] blue; [O I] magenta; $\mathrm{H} \alpha$ green; [N II] orange; [S II] red. Image credits. IC 4406: ESO/J. Richard (CRAL); NGC 6563: ESO/P. Weilbacher (AIP).

\section{MUSE Mapping of NGC 3132 and NGC 7009}

The medium ionization PN, NGC 7009 (Saturn Nebula), was selected as a Science Verification target for MUSE in 2014 primarily on account of its size, which fits inside the MUSE field of view, the very high emission line surface brightness, and the availability of a wealth of previous observations, including Hubble Space Telescope (HST) imaging [10] and deep spectroscopy [4,5]. NGC 3132 was selected from among the MUSE PN observations during early commissioning for detailed analysis; on account of its larger projected elliptical form (dimensions $\sim 60 \times 85^{\prime \prime}$ ), a mosaic of MUSE pointings was performed to cover the optical emission extent. The ionization level of NGC 3132 is moderate with weak He II emission, but the central object is a wide visual binary with an A-type central star companion to the hot post-AGBstar [11].

The data for both nebulae were similarly reduced and for NGC 3132 with larger offsets to cover a full field of $124 \times 60^{\prime \prime}$; the sky background was estimated from the corners of the field and with a position well offset from any nebula halo emission. Emission lines were extracted from as many spaxels as possible given a signal-to-noise criterion and fitting multiple Gaussian profiles (see [12,13]

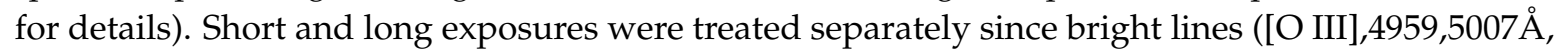
$\mathrm{H} \alpha$, etc.) were saturated on longer exposures, and even [O III]5007 $\AA$ was saturated over the bright shell emission in NGC 7009 in 10s exposure. The fitted emission line fluxes were reconstructed into images, and Figure 2 shows as an example the H $\beta$ and He II $5411 \AA$ images for NGC 7009, illustrating the difference in structure between low and high ionization species. (ESO Release eso1731 https:/ / www.eso.org/public/news/eso1731/) shows a multi-line color composite image from these data, which can be compared to the HST image (NASA opo9738g https: / / www.spacetelescope.org / images/opo9738g/). 

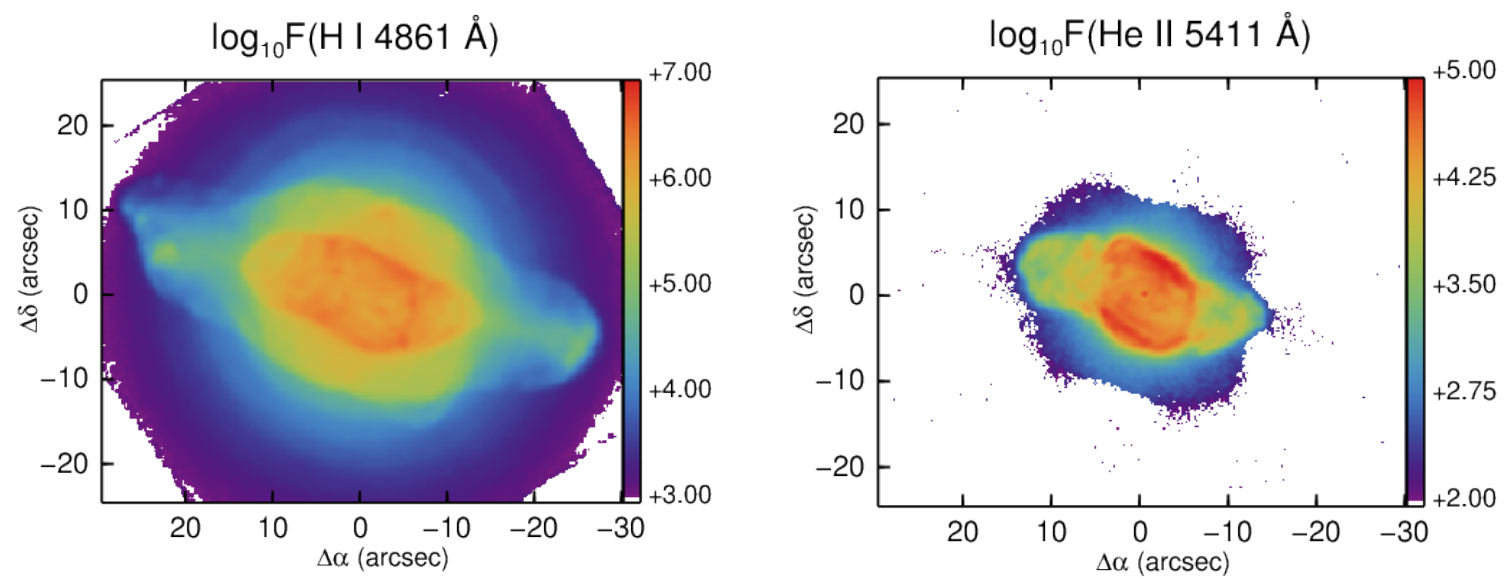

Figure 2. MUSE emission line images of NGC 7009: hydrogen Balmer H $\beta 4861 \AA$ (left) and He II $5411 \AA$ 7-4 line (right), from [12].

\subsection{Spectral Analysis: Line Ratios}

For both nebulae, a common analysis was performed, and the following maps were derived:

- reddening from a ratio of $\mathrm{H} \alpha$ to $\mathrm{H} \beta$ (and also Paschen lines in the red, such as P10 (9015 $\AA$ ) and P9 (9229 $\AA$ )) compared to Case B (nebula optically thick to HILyman- $\alpha$ photons; [14]);

- $\quad$ dereddened line maps (in absolute flux) for all measured lines;

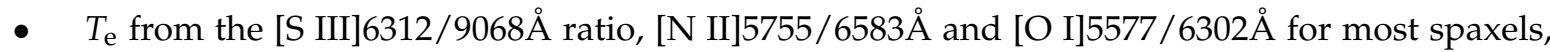
and from [Ar III]5192/7135 $\AA$ in brighter regions (PyNeb [15] was used for nebular diagnostic computations);

- $\quad N_{\mathrm{e}}$ from [Cl III] $5517 / 5537 \AA$ and [S II]6716/6731

- $\quad T_{\mathrm{e}}$ from He I line ratios together with $3889 \AA$ optical depth;

- $\quad N_{\mathrm{e}}$ from the ratio among the high series Paschen lines;

- $\quad T_{\mathrm{e}}$ from the magnitude of the Paschen jump at $8210 \AA$ with respect to HI P11 (8863 $\AA$ ) line strength;

- ionic abundances of many species using the appropriate $N_{\mathrm{e}}, T_{\mathrm{e}}$ diagnostics, such as $\mathrm{O}^{+}$and $\mathrm{O}^{++}$, $\mathrm{S}^{+}$and $\mathrm{S}^{++}$, and $\mathrm{Ar}^{++}$and $\mathrm{Ar}^{+++}$;

- $\quad$ total abundances of $\mathrm{He}$ and $\mathrm{O}$.

For both nebulae, although these diagnostics are standard, MUSE allows for the first time large field, high fidelity spatial mapping of their variation across the projected nebula surface.

Whilst it is difficult to compare distinct $\mathrm{PNe}$, with a sample of only two, and chosen with diverse criteria, several points of similarity, which arise directly from the IFU spectroscopy, can be selected by a comparison of the results presented in $[12,13,16]$ :

- The logarithmic extinction maps, $c(\mathrm{H} \beta)$, show distinct features over the nebulae pointing to the presence of dust within the ionized regions (see Figure 3 and [16]). Whilst not unexpected, since some evidence of spatial extinction has been observed in the optical for a few PNe to date and dust emission detectable in the infrared is almost ubiquitous (e.g., [17]), the association of dust with distinct features of the nebular structure, such as shell edges, knots, etc., is noteworthy;

- $T_{\mathrm{e}}$ measured from the ratio of He I lines, or from the magnitude of the Paschen continuum jump at $8210 \AA$, can be compared with the Collisionally Excited Line (CEL) $T_{\mathrm{e}}$ from the [S III] line ratio, to determine a CEL to recombination line temperature difference. In both NGC 7009 and NGC 3132, the difference in $T_{\mathrm{e}}$ increases towards the central star (see Figure 4). The radial trend of this increase is in the same sense as the increase of the Abundance Discrepancy Factor (ADF) measured between ORL and CEL abundances, for example for $\mathrm{O}^{+}$[18], although not yet in the same nebulae; 
- The maps of total $\mathrm{O}$ abundance (i.e., $\mathrm{O} / \mathrm{H}$ ), derived on various assumptions of Ionization Correction Factor (ICF) to correct for the fact that [O IV] emission is not observed in the optical, is not flat for NGC 3132 or NGC 7009 ([12,13], as would be expected from evolutionary considerations (short-term alterations in $\mathrm{O}$ are not predicted in the late stages of low mass star evolution). The deviations from flatness in the $\mathrm{O} / \mathrm{H}$ maps imply that the ICF procedure is incomplete: Figure 13 for NGC 3132 [13] and 21 for NGC 7009 [12] demonstrate that these O abundance modulations are correlated with the main shells and/or ionization zones.
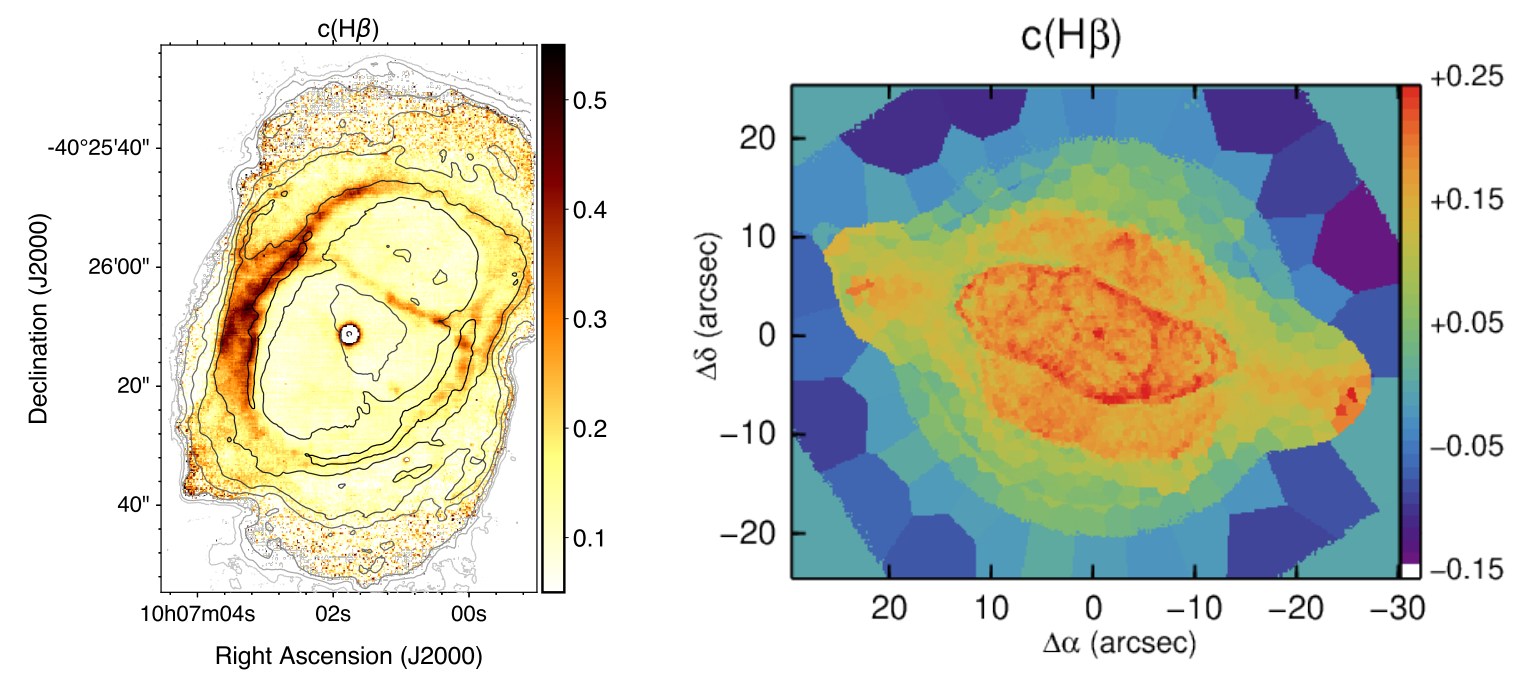

Figure 3. Comparison of the extinction, $c(\mathrm{H} \beta)$, maps of NGC 3132 (left, from [13]) and NGC 7009 (right, from [16]) showing strong evidence for internal dust structures obscuring background emission. The NGC 3132 image is produced in the native spaxel format, whilst the $c(\mathrm{H} \beta)$ image for NGC 7009 is produced from the ratio of $\mathrm{H} \alpha$ and $\mathrm{H} \beta$ images, which have been Voronoi tessellated to a constant signal-to-noise on $\mathrm{H} \beta$ flux.
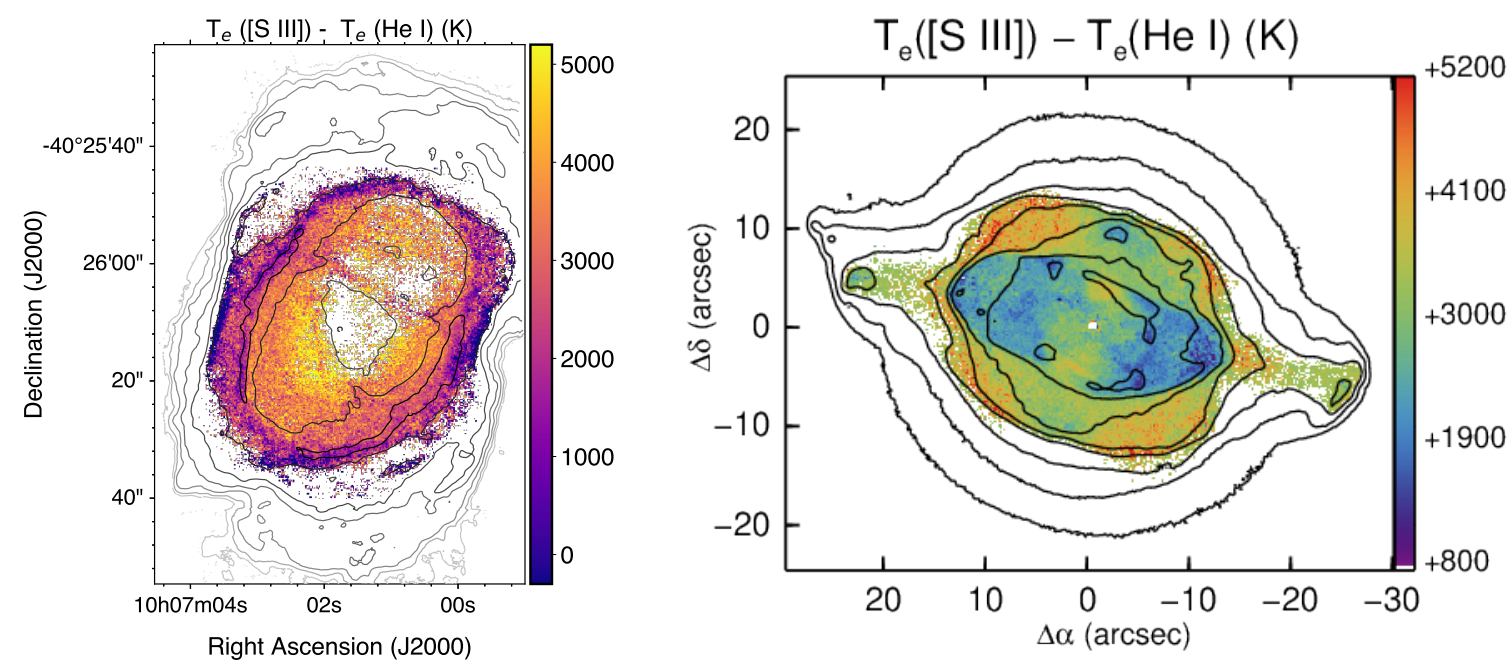

Figure 4. Comparison of the difference in $T_{\mathrm{e}}$ between the [S III] and He I $T_{\mathrm{e}}$ 's in NGC 3132 (left [13]) and NGC 7009 (right [12]). An increasing trend (larger difference between $T_{\mathrm{e}}$ from [S III] and He I) is found in both nebulae towards the location of the central star within the main shell.

\subsection{Spectral Analysis: Radial Velocities}

Emission line profiles in PNe are broadened locally by thermal and turbulent velocities and globally by the nebular expansion (typically $15-40 \mathrm{~km}^{-1}$ ); thus, the line profiles of metal lines have intrinsic widths of typically $10-20 \mathrm{kms}^{-1}$, while the $\mathrm{H}$ and $\mathrm{He}$ lines are broader. Although the 
resolution of MUSE spectra is intermediate, around $2.7 \AA$ (velocity resolution $170-85 \mathrm{kms}^{-1}$ from blue to red), some limited velocity information can be determined from Gaussian fitting of the lines. In [13], the velocity field of NGC 3132 was measured from several lines and showed a trend from higher to lower velocities from NW to SE; this trend was better seen in the forbidden lines and for the lower ionization lines of [N II] and [O I], but the behavior in the lower ionization species was more complex with both receding and approaching velocities in both lobes. A simple expanding shell seems difficult to reconcile with the pattern of velocities, and the velocity field could fit much better in a diabolo model, as proposed in [19].

\section{Prospects}

MUSE has great potential for moving spatial spectroscopic studies of PNe to a higher level, beyond the existing methods of slit-scanning and narrow band imaging, to high fidelity mapping of the full extent of the nebulae from the highest ionization regions near the central star to the neutral outer regions. There is also great potential for the discovery of additional domains in $\mathrm{PNe}$, such as the Low Ionization Structure (LIS) regions found in at the extremities of NGC 3132 [13], where a mix of photoionization and shock excitation and abundance imprints of distinct late-stage stellar ejecta may exist.

Given the already substantial set of PNe observed with MUSE (Table 2), covering a wide range in central star temperature, morphology, and ADF, comparative studies can be initiated to explore correlations between diagnostics. Such a database can enable improvements in the domain of different CEL ratios for $N_{\mathrm{e}}, T_{\mathrm{e}}$ measurement, with the impetus for improvement of atomic data where clear discrepancies arise. With a large set of empirical line ratios, diagnostics, and abundances, covering a diverse set of $\mathrm{PNe}$, improvements in ICF's are enabled with implications for all targets of nebular spectroscopy.

The intermediate spectral resolution and lack of blue response of MUSE (Section 2) will be compensated by BlueMUSE [20], with coverage to $3500 \AA$ at a resolving power of $>4000$, which is a proposed instrument for the VLT in the 2020s. BlueMUSE brings several strategic advantages for the study of PNe: the availability of the auroral [O III] $4363 \AA$ line for $T_{\mathrm{e}}$ measurement from the $4363 / 5007 \AA$ ratio for the bulk ionization stage of the dominant nebular coolant; coverage of the strong [O II] $3726,3729 \AA$ doublet for the $\mathrm{N}_{\mathrm{e}}$ and $\mathrm{O}^{+}$fraction; coverage and higher spectral resolution for measuring the weak $\mathrm{O}$ and $\mathrm{C}$ ORLs, enabling the spatial mapping of the ADF, at least for O.

Author Contributions: J.R.W. attended the WorkPlaNS II Lorentz Center workshop in Leiden, the Netherlands where this work was presented. J.R.W. and A.M.-I. each led one of the two projects described. All authors have read and agreed to the published version of the manuscript.

Funding: This research was partially funded by Spanish MINECO through Project AYA2015-68217-P.

Acknowledgments: We thank the referees for their comments, which helped to clarify the presentation. A.M.I. acknowledges support from the Spanish MINECO through Project AYA2015-68217-P.

Conflicts of Interest: The authors declare no conflict of interest.

\section{References}

1. Bacon, R.; Accardo, M.; Adjali, L.; Anwand, H.; Bauer, S.; Biswas, I.; Blaizot, J.; Boudon, D.; Brau-Nogue, S.; Brinchmann, J.; et al. The MUSE second-generation VLT instrument. Soc. Photo-Opt. Instrum. Eng. SPIE Conf. Ser. 2010, 7735, 773508. [CrossRef]

2. Richard, J.; Bacon, R. MUSE User Manual, Version 9.1; ESO: Garching, Germany, 2019.

3. Dopita, M.A.; Ali, A.; Sutherland, R.S.; Nicholls, D.C.; Amer, M.A. IFU spectroscopy of southern planetary nebulae IV: A physical model for IC 418. Mon. Not. R. Astron. Soc. 2017, 470, 839-864. [CrossRef]

4. Fang, X.; Liu, X.W. Very deep spectroscopy of the bright Saturn nebula NGC 7009-I. Observations and plasma diagnostics. Mon. Not. R. Astron. Soc. 2011, 415, 181-198. [CrossRef]

5. Fang, X.; Liu, X.W. Very deep spectroscopy of the bright Saturn nebula NGC 7009-II. Analysis of the rich optical recombination spectrum. Mon. Not. R. Astron. Soc. 2013, 429, 2791-2851. [CrossRef] 
6. García-Rojas, J.; Madonna, S.; Luridiana, V.; Sterling, N.C.; Morisset, C.; Delgado-Inglada, G.; Toribio San Cipriano, L. s-process enrichment in the planetary nebula NGC 3918. Results from deep echelle spectrophotometry. Mon. Not. R. Astron. Soc. 2015, 452, 2606-2640. [CrossRef]

7. Zhang, Y.; Liu, X.W.; Wesson, R.; Storey, P.J.; Liu, Y.; Danziger, I.J. Electron temperatures and densities of planetary nebulae determined from the nebular hydrogen recombination spectrum and temperature and density variations. Mon. Not. R. Astron. Soc. 2004, 351, 935-955. [CrossRef]

8. Peimbert, M.; Torres-Peimbert, S. Type I planetary nebulae. In Planetary Nebulae; Aller, L.H., Ed.; IAU Symposium; Reidel: Dordrecht, The Netherlands, , 1983; Volume 103, pp. 233-242.

9. Cox, P.; Huggins, P.J.; Bachiller, R.; Forveille, T. CO in the southern planetary nebulae NGC 6072, NGC 6563, and IC 4406. Astron. Astrophys. 1991, 250, 533.

10. Balick, B.; Alexander, J.; Hajian, A.R.; Terzian, Y.; Perinotto, M.; Patriarchi, P. FLIERs and Other Microstructures in Planetary Nebulae. IV. Images of Elliptical PNs from the Hubble Space Telescope. Astron. J. 1998, 116, 360-371. [CrossRef]

11. Ciardullo, R.; Bond, H.E.; Sipior, M.S.; Fullton, L.K.; Zhang, C.Y.; Schaefer, K.G. A HUBBLE SPACE TELESCOPE Survey for Resolved Companions of Planetary Nebula Nuclei. Astron. Astrophys. 1999, 118, 488-508. [CrossRef]

12. Walsh, J.R.; Monreal-Ibero, A.; Barlow, M.J.; Ueta, T.; Wesson, R.; Zijlstra, A.A.; Kimeswenger, S.; Leal-Ferreira, M.L.; Otsuka, M. An imaging spectroscopic survey of the planetary nebula NGC 7009 with MUSE. Astron. Astrophys. 2018, 620, A169. [CrossRef]

13. Monreal-Ibero, A.; Walsh, J.R. The MUSE view of the planetary nebula NGC 3132. Astron. Astrophys. 2020, 634, A47. [CrossRef]

14. Osterbrock, D.E.; Ferland, G.J. Astrophysics of Gaseous Nebulae and Active Galactic Nuclei; University Science Books: Sausalito, CA, USA, 2006.

15. Luridiana, V.; Morisset, C.; Shaw, R.A. PyNeb: A new tool for analyzing emission lines. I. Code description and validation of results. Astron. Astrophys. 2015, 573, A42. [CrossRef]

16. Walsh, J.R.; Monreal-Ibero, A.; Barlow, M.J.; Ueta, T.; Wesson, R.; Zijlstra, A.A. The extinction and dust-to-gas structure of the planetary nebula NGC 7009 observed with MUSE. Astron. Astrophys. 2016, 588, A106. [CrossRef]

17. Bernard-Salas, J. Infrared spectroscopy of planetary nebulae, including Spitzer. In Planetary Nebulae in our Galaxy and Beyond; Barlow, M.J., Méndez, R.H., Eds.; IAU Symposium; Cambridge University Press: Cambridge, UK, 2006; Volume 234, pp. 181-188. [CrossRef]

18. Wesson, R.; Jones, D.; García-Rojas, J.; Boffin, H.M.J.; Corradi, R.L.M. Confirmation of the link between central star binarity and extreme abundance discrepancy factors in planetary nebulae. Mon. Not. R. Astron. Soc. 2018, 480, 4589-4613. [CrossRef]

19. Monteiro, H.; Morisset, C.; Gruenwald, R.; Viegas, S.M. Morphology and Kinematics of Planetary Nebulae. II. A Diabolo Model for NGC 3132. Astrophys. J. 2000, 537, 853-860. [CrossRef]

20. Richard, J.; Bacon, R.; Blaizot, J.; Boissier, S.; Boselli, A.; Bouché, N.; Brinchmann, J.; Castro, N.; Ciesla, L.; Crowther, P. BlueMUSE: Project Overview and Science Cases. arXiv 2019, arXiv:1906.01657.

(C) 2020 by the authors. Licensee MDPI, Basel, Switzerland. This article is an open access article distributed under the terms and conditions of the Creative Commons Attribution (CC BY) license (http:// creativecommons.org/licenses/by/4.0/). 